\title{
Fundamentos teóricos a problemas clínicos en los trastornos de la personalidad y su proyección en el DSM-5/CIE- 11
}

\author{
Ana Rodríguez Rosado, ${ }^{1}$ Andrés Fontalba Navas, ${ }^{2}$ José Miguel Pena Andreu'
}

Artículo original

\section{SUMMARY}

More research needs to be carried out into the theoretical backgrounds of the various specialities in Medicine, and particularly in Psychiatry. Since new classifications for mental disorders are soon to be introduced, we will briefly examine the history of personality disorders and our interest will be directed to problems we might encounter using the current diagnostic criteria, i. e., DSM-IV/ICD-10.

Key words: Personality disorders, historical background, diagnostic criteria.

La necesidad de profundizar en los fundamentos teóricos de la práctica clínica no es algo exclusivo de la Psiquiatría: cualquier facultativo en Medicina se ve obligado a revisar con frecuencia los aspectos teóricos de su especialidad. En este sentido, se advierte con facilidad el peligro que genera la posibilidad de dejarse arrastrar por la atención al enfermo sin reparar en las cuestiones conceptuales, que son las que, en último término, permiten realizar con acierto las acciones relativas a la evaluación y el tratamiento.

En este trabajo realizamos, en primer lugar, una breve revisión de la historia de los trastornos de personalidad, orientada a una mayor comprensión de los mismos desde el punto de vista asistencial, y nos centramos, después, en los actuales criterios diagnósticos ${ }^{1,2}$ analizando algunos aspectos relacionados con su aplicación en la práctica clínica diaria.

Los distintos estudios en los que se revisa la historia de los trastornos de personalidad coinciden en señalar a Hipócrates (s. V a. C.) como el primer autor de obligada referencia a la hora de analizar las relaciones entre lo psíquico y la corporalidad. Con su descripción de los cuatro tipos de temperamentos (melancólico, sanguíneo, colérico y flemático), en función de la proporción relativa de los cuatro humores corporales (bilis negra, sangre, bilis amarilla y flema, respectivamente), no estaba lejos del punto de partida de una medicina científica. No hay que olvidar, sin embargo, que la medicina hipocrática enfatiza la salud como un todo y el valor de la aproximación al paciente como un ser humano completo. ${ }^{3}$

Aunque hay diversos sistemas tipológicos, como el de Sigaud, que representa la escuela francesa de biotipología, para la que el biotipo resultaría de la huella que el medio ambiente imprime al soma, sin intervención de la herencia ni de factores constitucionales; o el de Viola y Pende, pertenecientes a la escuela italiana, que busca la génesis del biotipo en las leyes biológicas, ${ }^{4}$ son los biotipos de Kretschmer y Sheldon los que se siguen usando con mayor frecuencia en la actualidad.

Ernst Kretschmer, al estudiar la relación entre los caracteres morfológicos y las propiedades psicológicas, distingue tres tipos básicos de estructura corporal que son los biotipos pícnico, leptosómico y atlético. Los tipos de estructura corporal se relacionan con los tipos de carácter: el temperamento ciclotímico, el esquizotímico y el viscoso. ${ }^{5}$ Su escuela propone una relación de transición entre personalidad y enferme-

Departamento de Psiquiatría y Fisioterapia. Facultad de Medicina. Universidad de Málaga, España

2 Unidad Gestión Clínica Salud Mental. Area Gestión Sanitaria Norte de Almería, España.

Correspondencia: Andrés Fontalba Navas. Unidad Salud Mental Comuniataria Huercal-Overa. C/Silvestre Martinez de Haro SN. 04600 Huercal-Overa (Almería), España. E-mail: andresfontalba@gmail.com

Recibido primera versión: 22 de enero de 2012. Segunda versión: 2 de octubre de 2012. Aceptado: 9 de octubre de 2012 
dad mental, como señalaremos a continuación al revisar propiamente la historia de los trastornos de personalidad. Sus estudios fueron un punto de partida para las investigaciones de William Herbert Sheldon que encabeza la escuela anglosajona y, posteriormente, las de Conrad y Janzarik. ${ }^{4}$

Dejando aparte las hipótesis constitucionalistas, que agruparían a los individuos en torno a pocos tipos de rasgos físicos y psíquicos característicos, la historia moderna de los trastornos de personalidad se sitúa a principios del siglo XIX, cuando psiquiatras como Pinel, Morel o Pritchard, entre otros, describieron algunos tipos de personalidad con dificultades de adaptación social que encontraban en la práctica clínica. Se trataría de sujetos afectos de lo que calificaron como "manía sin delirio" (Pinel), "locura de los degenerados" (Morel) o "locura moral" (Pritchard), en los cuales se daba una perversión de los sentimientos, de los hábitos morales y de los impulsos, sin experimentar ningún defecto de la inteligencia o la capacidad de razonamiento, y sin la presencia de alucinaciones. Estos tipos corresponderían a lo que actualmente conocemos como "personalidades psicopáticas", uno de los grandes bloques de la patología del carácter.

A comienzos del siglo $X X$, y desde una concepción psicogénica o psicodinámica, Janet y Freud (y Charcot con anterioridad), estudiaron el modelo psicológico de la histeria, entidad que antecede al trastorno histriónico de la personalidad. En este contexto la escuela psicoanalítica describió con el nombre de "neurosis de carácter" a las organizaciones de la personalidad fijadas a la estructura característica de una fase del desarrollo libidinal (así se describe el carácter sadomasoquista o el obsesivo-compulsivo). Posteriormente, y ya en el marco de las relaciones objetales, la teoría psicoanalítica propone que la personalidad se forma principalmente en la primera infancia a partir de las relaciones parentales. ${ }^{6}$

Dentro de este contexto cabe destacar, por sus repercusiones clínicas, las características del Yo neurótico. Es probable que tengamos experiencia clínica acerca de la valoración de pacientes, generalmente mujeres, con una extrema sensibilidad emocional y rasgos de marcada inseguridad, que hacen difícil la relación terapéutica (y las relaciones interpersonales en general).

Como describe Henri Ey al hablar de la semiología de los trastornos de la personalidad, el Yo neurótico es un Yo que no alcanza a resolver el conflicto interno de su identificación: yo no soy "yo" sino por la identificación a "un personaje" que constituye el ideal de mi Yo. Pues bien, el neurótico "no consigue asumir el papel de su personaje, identificarse consigo mismo y autentificar su persona; y es en una especie de juego artificial, en un sentido falso, como el neurótico vive su angustia". ${ }^{7}$ Al no resolver el problema interno de su identificación, las relaciones consigo mismo y con los demás se ven afectadas: el malestar del individuo frente a sí mismo $\mathrm{y}$ a los otros es experimentado como una angustia en la que se mezclan los sentimientos de culpabilidad, el deseo de castigarse, la decepción ante las frustraciones, los complejos de inferioridad, etc. Además, a pesar de las apariencias, la angustia del paciente neurótico no depende de las circunstancias sino que es interna e inconsciente. Estos son algunos de los aspectos clínicos que suelen considerarse característicos de los pacientes neuróticos.

Desde una perspectiva distinta, en la década de 1920 autores de la escuela alemana, encabezada por Kretschmer, describieron los tipos de la personalidad como formas de transición de las psicosis paranoides y afectivas. ${ }^{5}$ Estas variantes de la personalidad se corresponderían con los actuales trastornos paranoide, esquizotípico o ciclotímico de la personalidad, según los criterios del DSM-IV (la CIE-10 no incluye el trastorno esquizotípico ni el ciclotímico en el apartado de "Trastornos de personalidad" sino en el bloque de "Esquizofrenia, trastorno esquizotípico y trastornos de ideas delirantes" y en el de "Trastornos del humor", respectivamente). La interconexión entre personalidad y trastorno psicopatológico es uno de los campos de mayor interés que continúa abierto a la investigación. ${ }^{8}$

El fenomenólogo alemán Kurt Schneider, por el contrario, consideró que los trastornos de personalidad representaban desviaciones que excedían los límites de los tipos medios de variantes personales, dando lugar a tipos clínicos bien definidos. Por tanto, no contempló los trastornos de personalidad como precursores de otros trastornos mentales, incluso más severos, sino como entidades coexistentes (en esta línea las clasificaciones americanas a partir del DSM-III, que se publica en 1980, sitúan los trastornos de personalidad en un eje distinto al de las enfermedades mentales). Desarrolló además un sistema amplio de categorías de los trastornos de personalidad que proporcionó el modelo para muchos de los trastornos contemplados en las clasificaciones actuales. No obstante, el propio Schneider señaló también la dificultad de hacer una descripción plástica de los tipos psicopáticos. ${ }^{9}$

En todo caso, a pesar de que la dicotomía entre lo categorial y lo dimensional continúa abierta, el sustrato teórico de las categorías diagnósticas para los trastornos de personalidad ha ido cambiando con el paso del tiempo, y las descripciones actuales intentan representar una síntesis entre la tradición clínica y los hallazgos empíricos. ${ }^{10,11}$

Es bueno que los clínicos profundicemos en los fundamentos teóricos de nuestra especialidad, porque la reflexión y la discusión sobre la naturaleza de los trastornos psicopatológicos redunda en beneficio de la práctica clínica y facilita el desarrollo de la investigación.

Los pacientes con trastornos de personalidad representan uno de los principales retos para los clínicos, puesto que obligan al terapeuta a poner en marcha con la máxima intensidad sus propios recursos personales: ¿cómo tratar a pacientes que, por lo general, coinciden en su dificultad para 
relacionarse adecuadamente con los demás y cuya forma habitual de reaccionar ante las demandas del entorno suele agravar aún más esas dificultades? Además, pueden no reconocer que el origen de sus problemas resida en su propia personalidad, por lo que tienden a hacer responsables a los otros de lo que les sucede, e incluso pueden negar durante un tiempo la existencia de problema alguno. La complejidad en todos estos casos se ve aumentada porque lo que caracteriza a los pacientes con trastornos de personalidad no es sólo un problema de la persona sino algo que afecta al eje de lo que dicha persona es. De este modo, la patología mental, y en concreto los trastornos de personalidad, a diferencia de la patología general, aparece ante nosotros "no como una patología de la vida orgánica que amenaza más o menos mortalmente la 'vida', sino como una patología de la vida psíquica que amenaza al hombre en su humanidad". ${ }^{7}$

A la hora de hacer el abordaje diagnóstico en la práctica clínica encontramos una serie de cuestiones que conviene analizar. Por ejemplo, la CIE-10 considera que estos trastornos abarcan formas de comportamiento duraderas y profundamente arraigadas en el enfermo, que se manifiestan como modalidades estables de respuesta a un amplio espectro de situaciones individuales y sociales. ${ }^{2}$ Por su parte, el DSM-IV considera los trastornos de personalidad como patrones de rasgos inflexibles y desadaptativos que provocan malestar subjetivo, deterioro social o laboral significativo, o ambas cosas. ${ }^{1}$ Ahora bien, contemplar los trastornos de personalidad como patrones crónicos y estables lleva consigo ciertas limitaciones. De hecho, ¿hasta qué punto un rasgo de personalidad puede considerarse inflexible? Todos tenemos experiencia de cómo pueden modificarse algunos rasgos de personalidad que considerábamos escasamente susceptibles de cambio. En este sentido puede hablarse de una "elaboración personal del carácter" o "formación de la personalidad" en la línea de lo que queremos llegar a ser, si bien esa elaboración no se lleva a cabo de forma automática o espontánea: requiere el concurso de nuestra libertad. Además, si esos rasgos son inmodificables o invariables, ¿por qué tratamos de modificarlos en la relación terapéutica? Por tanto, cabe pensar que, también en los trastornos de la personalidad, existe un espacio interior más o menos accesible a las orientaciones del terapeuta y a las exigencias externas. No equivale el concepto de trastorno de la personalidad a una imposibilidad de alcanzar una maduración personal.

Por tanto podemos hablar de rasgos de personalidad "invariables" en el sentido de que son lo suficientemente estables como para identificar el hábito, el estilo de reactividad de cada uno, pero sin olvidar que el sistema de personalidad es precisamente el Yo en tanto que "dueño de su carácter, actor de su personaje, artesano de su mundo y sujeto de su conocimiento", 7 y en este sentido siempre podemos introducir cambios que modifiquen ese estilo de reacción que está impidiendo un desarrollo adecuado del Yo.
Estos rasgos, continúa diciendo la CIE-10, ${ }^{2}$ representan desviaciones extremas, o al menos significativas, del modo como el individuo normal de una cultura percibe, piensa, siente $\mathrm{y}$, sobre todo, se relaciona con los demás, o deben apartarse acusadamente de las expectativas culturales o norma, de acuerdo con el DSM-IV. ${ }^{1}$ Esta última clasificación también señala que la desviación debe ponerse de manifiesto en más de una de las siguientes áreas: cognitiva, afectiva, de control de impulsos, de necesidad de gratificación y de forma de relacionarse con los demás. ${ }^{1}$

Conviene precisar aquí que la "desviación extrema o significativa" o la "norma" a la que hacen referencia los Manuales, no ha de interpretarse en sentido cuantitativo (desviaciones de la media estadística): una persona no tiene un trastorno de personalidad en función de la intensidad de sus vivencias intelectivas, afectivas, etc. No se trata de una cuestión cuantitativa sino de valorar hasta qué punto hay en ese individuo una dificultad real para comportarse de una forma adecuada. Y esto puede apreciarse mediante la relación interpersonal que se establece en la entrevista clínica, la cual es el instrumento por excelencia para el diagnóstico y para la conceptualización del trastorno mental. De ahí que lo que debemos preguntarnos no es tanto hasta qué punto las conductas de una persona son extremas desde el punto de vista cuantitativo, sino hasta qué punto su comportamiento es consecuencia de una limitación previa en su personalidad que compromete sus posibilidades de completar satisfactoriamente su desarrollo como persona.

Además, a la hora de hacer un diagnóstico de trastorno de personalidad, no sólo hemos de comprobar una conducta socialmente desajustada, sino también malestar clínicamente significativo o deterioro del funcionamiento social, laboral o a nivel de otras áreas importantes de la actividad del sujeto. ${ }^{1,2}$ De hecho, si no hubiera un deterioro funcional podría hablarse de un conjunto de síntomas pero no debería utilizarse el término "trastorno" (cuestión distinta sería la de cómo medir el deterioro funcional). ${ }^{11}$

Por último, tanto la CIE-10 como el DSM-IV coinciden también en el criterio temporal: la desviación debe haberse establecido de forma crónica desde la adolescencia o al principio de la edad adulta y ser persistente. Es decir, debe manifestarse en una amplia gama de situaciones más que en una situación desencadenante concreta o en respuesta a un estímulo particular. Asimismo se requiere que la alteración de la personalidad no sea atribuible a una lesión o enfermedad cerebral o a otros trastornos psiquiátricos.

Estas breves reflexiones, y muchas otras que podrían hacerse, nos remiten de nuevo al pensamiento de Henri Ey, el cual, después de decir que para el psiquiatra ocupado de la clínica las teorías han pasado al segundo plano de sus preocupaciones, insiste en que, en todo caso, no puede evitar el referirse a un esquema teórico que le obliga, incluso a pesar suyo, a tomar posición con respecto a los problemas difíciles en los cuales compromete necesariamente su concepción y su 
terapéutica de la enfermedad mental. Es decir, que en definitiva "está obligado a adoptar una cierta manera de juzgar o de prejuzgar las relaciones entre lo físico y lo moral, entre el cerebro y el pensamiento, entre la constitución de la persona y el medio, etc". ${ }^{7}$ La posición teórica de cada uno vendrá determinada por la escuela a la que pertenezca o con la que más se identifique, ya sea psicodinámica, biomédica o sociocultural.

Las definiciones y criterios sobre los trastornos de la personalidad pueden servirnos más o menos, pero lo importante es que los clínicos seamos capaces de identificar esos trastornos en nuestros pacientes. Primero, porque son personas que necesitan una ayuda especializada (a diferencia de otros problemas de salud mental que pueden ser atendidos en el ámbito de la atención primaria), un "plan individualizado de tratamiento", que diríamos utilizando el lenguaje actual de los sistemas sanitarios públicos; segundo, porque tener un trastorno de la personalidad no sólo genera problemas en la persona que lo padece sino en el entorno familiar, laboral y social (incluso con consecuencias legales); tercero, porque son trastornos que muchas veces interfieren en la evolución de otro trastorno mental coexistente, bien sea de tipo afectivo, alimentario, adictivo, etc. De hecho, se observa que los trastornos afectivos son frecuentes en los trastornos de personalidad, por lo que tendría interés explorar la posible comorbilidad, ya que ésta comporta implicaciones importantes en el tratamiento y en los resultados globales de cualquier abordaje terapéutico. ${ }^{12}$

Con respecto a las futuras clasificaciones internacionales de enfermedades y las tendencias que se atisban en sus planteamientos, el debate actual se centra en la posibilidad de mejorar la representación de los trastornos de personalidad en el DSM-5 y en la CIE-11. Como se mencionó anteriormente, la discusión acerca de si los trastornos de la personalidad están mejor clasificados como dimensiones o cómo categorías permanece abierta: ¿se describen mejor los trastornos de personalidad como representaciones dimensionales de categorías diagnósticas o extremos dimensionales del funcionamiento general de la personalidad, o como representaciones categoriales en sí mismas?

Aunque, ciertamente, los modelos categóricos aplicados a los trastornos de personalidad han facilitado un lenguaje común y han servido para un uso generalizado en investigación, también son conocidas sus limitaciones: el solapamiento entre los diagnósticos, la gran heterogeneidad dentro de los pacientes que reciben el mismo diagnóstico, los límites arbitrarios entre el funcionamiento normal y patológico de la personalidad, y la dificultad para abarcar las distintas manifestaciones psicopatológicas, de modo que el diagnóstico de trastorno de personalidad "no especificado" es el más común.

Estas dificultades hacen que sea necesario clarificar si los trastornos mentales en general, y los trastornos de personalidad en particular, no estarían mejor representados en forma de dimensiones psicopatológicas que mediante categorías múltiples. ${ }^{13}$ De hecho, algunos autores no dudan en afirmar la necesidad de desarrollar modelos dimensionales de diagnóstico, ${ }^{14}$ y distintos modelos dimensionales se ofrecen como solución a los problemas que plantea el diagnóstico categorial. ${ }^{11}$

La mayoría de los clínicos, sin embargo, no están familiarizados con los modelos dimensionales sino con el modelo médico, donde un único diagnóstico sirve para comunicar gran cantidad de información sobre los problemas del paciente, el tratamiento y el pronóstico más probable. En todo caso, los defensores del modelo dimensional señalan que algunos fenómenos clínicos en medicina, tal como la presión arterial, tienen una distribución continua aunque conducen a un diagnóstico categorial (por ejemplo, hipertensión), una vez que se establecen los puntos de corte compatibles con enfermedad y necesidad de tratamiento.

La bibliografía publicada en relación con el futuro de las clasificaciones de los trastornos de la personalidad no descarta que los próximos manuales diagnósticos se basen en un modelo híbrido que incluya los siguientes aspectos: 1. una valoración global del funcionamiento de la personalidad que abarque desde lo que se considera normal hasta lo gravemente deteriorado; 2 . descripciones prototípicas de los principales trastornos de personalidad; 3 . un examen de rasgos de personalidad basado en prototipos, pero que pueda ser usado también para describir las principales características de pacientes que, o bien no tienen un trastorno de personalidad, o tienen un trastorno de personalidad que no se ajusta a ninguno de los prototipos; 4 . criterios generales de trastorno de personalidad que contemplen el déficit en la diferenciación e integración del Yo y en la capacidad para establecer relaciones interpersonales; 5 . medidas de un funcionamiento adaptativo o adecuado de la persona. ${ }^{15}$

Con independencia de que la investigación sobre estos temas pueda modificar en mayor o menor medida las previsiones, se observa una tendencia a sustituir los modelos clasificatorios categoriales actuales (DSM-IV, CIE-10), basados en una psicopatología descriptiva de los trastornos mentales (listados de síntomas), por modelos dimensionales o mixtos, que incluyan, entre otras medidas, valoraciones de los rasgos normales de la personalidad, dando lugar así a aproximaciones más amplias que permitan una mayor comprensión de la psicopatología de la personalidad.

\section{REFERENCIAS}

1. DSM IV-TR, Breviario, criterios diagnósticos. Barcelona: Masson; 2003.

2. CIE 10, Décima revisión de la clasificación internacional de las enfermedades. Trastornos mentales y del comportamiento. Madrid: Meditor; 1992.

3. Mezzich JE. La base dialogal de nuestra profesión: Psiquiatría con la persona. World Psychiatry 2007;(edición especial)5(3):129-130.

4. Ruiz Ruiz M. La estructura científica de la psicopatología Segunda edición. Málaga, España: Cepyp; 1989.

5. Jaspers K. Psicopatología general. Cuarta edición. Editorial Buenos Aires: Beta; 1973.

6. Philips KA, Gunderson TG. Tratado de psiquiatría. Tercera edición. Tomo I, p. 794, Hales: Masson; 2000. 
7. Ey H, Bernard P, Brisset Ch. Tratado de psiquiatría Octava edición. Barcelona: Masso; 1992.

8. Widiger TA, Simonsen E. Alternative dimensional models of personality disorder: finding a common ground. J Pers Disord 2005;19:110130.

9. López Ibor JJ. Las neurosis como enfermedades del ánimo. Madrid: Editorial Gredos; 1966

10. Coolidge FL, Segal DL. Evolution of personality disorder diagnosis in the diagnostic and statistical manual of mental disorders. Clinical Psychology Review 1998;18(5):585-599.

11. Widiger TA, Simonsen E, Krueger R, Livesley WJ et al. Personality disorder research agenda for the DSM-V. J Pers Disord 2005;19:315-338.
12. Vilaplana M, Mc Kenney C, Riesco MD, Autonell J et al. Comorbilidad depresiva en los trastornos de la personalidad. Rev Psiquiatr Salud Ment (Barc.) 2010;3(1):4-12.

13. Kupfer DJ, First MB, Regier DA. Introduction. En: A research agenda for DSM-V. Kupfer DJ, First MB, Regier DA (eds.). Washington DC: American Psychiatric Association; 2002; pp. xv-xxiii.

14. Rounsaville BJ, Alarcón RD, Andrews G, Jackson JS et al. Basic nomenclature issues for DSM-V. En: A research agenda for DSM-V. Kupfer DJ, First MB, Regier DA (eds.). Washington DC: American Psychiatric Association; 2002; pp. 1-29.

15. Skodol AE, Bender DS. The future of personality disorders in DSMV? Am J Psychiatry 2009;166(4):388-391.

Artículo sin conflicto de intereses 